\title{
Fascicular shifting: a novel technique to overcome large nerve defects
}

\author{
Maria Hader, MD, ${ }^{1}$ Matthias E. Sporer, MD, ${ }^{1}$ Aidan D. Roche, PhD, ${ }^{1}$ Ewald Unger, ${ }^{3}$ \\ Konstantin D. Bergmeister, MD, ${ }^{1}$ Robert Wakolbinger, MD, ${ }^{1}$ and Oskar C. Aszmann, MD, PhD1,2 \\ ${ }^{1}$ Christian Doppler Laboratory for Restoration of Extremity Function; ${ }^{2}$ Division of Plastic and Reconstructive Surgery, Department \\ of Surgery; and ${ }^{3}$ Centre for Medical Physics and Biomedical Engineering, Medical University of Vienna, Austria
}

\begin{abstract}
OBJECTIVE Over the last decade, a number of authors have investigated the utility of different biological and synthetic matrices as alternatives to conventional nerve grafts. However, the autologous nerve graft remains the gold standard, even though it often involves using a pure sensory nerve to reconstruct a mixed or even a pure motor nerve. Furthermore, limited donor sites often necessitate a significant mismatch of needed nerve tissue, especially for large proximal nerve defects such as brachial plexus lesions. Here, the authors present a new technique that overcomes these problems: the fascicular shift procedure (FSP). A fascicular group of the nerve distal to the injury is harvested in a sufficient length to bridge the nerve defect.
\end{abstract}

METHODS The method of fascicular shifting was tested at the sciatic nerve in 45 Lewis rats. In the experimental group, a 15-mm nerve defect was created and reconstructed with a fascicular group that was harvested directly distal to the gap. This group was compared with 1 negative control group (defect without reconstruction) and 3 positive control groups (sensory, motor, and mixed graft). After 12 weeks of nerve regeneration, outcome was evaluated using retrograde labeling, histomorphometric analysis, and muscle force analysis.

RESULTS All reconstructed groups showed successful regeneration with various levels of function. The negative control group showed minimal force measurements that were of no functional value. The fascicular shift provided sufficient guidance to overcome nerve defects, had higher $(p<0.1)$ motor neuron counts $(1958.75 \pm 657.21)$ than the sensory graft (1263.50 \pm 538.90$)$, and was equal to motor grafts (1490.43 \pm 794.80$)$ and mixed grafts $(1720.00 \pm 866.421)$. This tendency of improved motor regeneration was confirmed in all analyses. The mixed graft group was compared with the experimental group to investigate the influence of the potential damage induced by the fascicular shift distal to the repair site. However, none of the analyses revealed an impairment of nerve regeneration for both the tibial and common peroneal index muscles.

CONCLUSIONS This study demonstrates that harvesting a transplant from the nerve segment distal to the injury site offers a mixed graft without causing additional donor-site morbidity. These grafts perform statistically better than a standard sensory graft in terms of motor recovery. The fascicular shift presents a novel method to reconstruct large proximal nerve defects, making it immensely attractive in brachial plexus reconstruction.

https://thejns.org/doi/abs/10.3171/2017.3.SPINE16276

KEY WORDS peripheral nerve defect; axonal regeneration; nerve graft; nerve reconstruction; surgery; animal model

$\mathrm{L}$ ARGE proximal nerve defects present a major challenge in the field of peripheral nerve reconstruction. Over the last decade, a number of investigators have studied the usefulness of different biological or synthetic matrices as alternatives to conventional nerve grafts; however, these techniques have limited indications and do not provide regenerative support over longer distances. , $^{8,31,34}$
One problem may be that a growth-promoting environment includes not only physical cues but also a rich spectrum of different growth factors provided by reactive Schwann cells and migrated macrophages. ${ }^{18,24,35}$

Thus, autologous sensory nerve transplantation is still the gold standard in the reconstruction of large peripheral nerve defects. In addition to problems such as donor-site

ABBREVIATIONS BW = body weight; $\mathrm{CP}=$ common peroneal nerve; $\mathrm{FSP}=$ fascicular shift procedure; $\mathrm{PFA}=$ paraformaldehyde; $\mathrm{TIB}=$ tibial nerve.

SUBMITTED March 5, 2016. ACCEPTED March 16, 2017.

INCLUDE WHEN CITING Published online October 6, 2017; DOI: 10.3171/2017.3.SPINE16276. 
morbidity and limited availability of grafting material, the functional outcome of this technique is frequently disappointing. A poor outcome may be due to a significant mismatch between axonal numbers, considering that one sural nerve provides only around 5600 mostly small-diameter axons. ${ }^{5}$ In proximal nerve lesions, this deficit is most critical given the immense number of nerve fibers that need to be reconstructed. Furthermore, deficient regeneration is believed to be the consequence of a qualitative mismatch in which a sensory graft is used to reconstruct a mixed sensory/motor nerve. It has been hypothesized that robust regeneration of motor axons, especially over long distances, needs motor pathways with adequate epitopes and humoral support. ${ }^{1-4,22}$ Obviously, this reconstructive goal is difficult to achieve since only a few nerve grafts in the sensory system can be spared, and the situation is even more critical in the motor system. ${ }^{31}$

In this report, we present an alternative method of nerve grafting that may overcome this problem: the fascicular shift procedure (FSP). Here, a fascicular group, containing both motor and sensory axons, of the nerve segment distal to the injury site is used to bridge the defect. This procedure has the advantage of "like-with-like" grafting without causing donor-site morbidity and addresses the problem of limited availability of grafting material. The purpose of this animal study was to determine whether the FSP represents a feasible and safe method of nerve grafting and, if so, whether the regeneration outcome is even superior to that of a standard sensory nerve graft.

\section{Methods \\ Animals}

Fifty-five male Lewis rats (Charles River) weighing approximately $250 \mathrm{~g}$ were used in this study, following the guidelines of our ethics committee. The animals were maintained on standard rat chow (Ssniff) and water ad libitum with a 12-hour light-dark cycle and standardized housing conditions. Each cage (size Type IV) housed a maximum of 4 rats. All animals received humane care in compliance with the principles of laboratory animal care as recommended by the Federation of European Laboratory Animal Science Associations (FELASA). ${ }^{13}$ Prior to the study, approval was obtained from the ethics committee of the Medical University of Vienna and the Austrian Federal Ministry of Science and Research (Bundesministerium für Wissenschaft, Forschung und Wirtschaft [BMWFW]).

Each intervention was performed with the animal under general anesthesia. Anesthesia was induced using 1 $\mathrm{ml} / \mathrm{kg} \mathrm{BW}$ of Ketasol (ketamine) and $0.05 \mathrm{ml}$ of Rompun (xylazine) administered intraperitoneally and maintained with $1.5 \%$ isoflurane inhalation. The animals were shaved and washed with an antiseptic solution after positioning for surgery. All surgical procedures were done under sterile conditions. Animals recovered in a warm environment. Preoperative pain was managed with Dipidolor injections ( $3 \mathrm{mg} / \mathrm{kg} \mathrm{BW}$ of piritramide, subcutaneously). For pain relief on the initial postoperative days, the drinking water was mixed with Dipidolor and glucose (2 ampoules of Dipidolor [ $=30 \mathrm{mg}$ of piritramide] $+10 \mathrm{ml}$ of $10 \%$ glucose solution in $250 \mathrm{ml}$ of drinking water).

\section{Experimental Outline}

Five groups were established (Fig. 1): One negative control group (A) and 3 positive control groups (B, C, D) were compared against the experimental group (E). In all groups, a critical nerve defect of $15 \mathrm{~mm}$ was created. In Group A, there was no repair. In Groups B, C, and D, the defect was reconstructed with a mixed, sensory, or motor nerve graft, respectively. In Group E, the nerve defect was reconstructed with a fascicular group harvested directly distal to the defect. To minimize the strain on animals in the positive control groups, we obtained the nerve grafts from 10 donor animals.

The nerves were allowed 12 weeks for regeneration. This time period guaranteed full recovery. ${ }^{18,38}$ The animals were then reanesthetized so that we could perform force measurements and retrograde double labeling to quantify regenerated axons after nerve repair (Fig. 2). Force measurements were performed to evaluate functional outcome. Directly after the force measurements, the tibial nerve (TIB) and common peroneal nerve (CP) were transected distal to the reconstruction site with the proximal end inserted into either Fluoro-Ruby or Fluoro-Gold solution (Fluorochrome LLC), respectively (this alternated between animals to avoid sampling bias). At the same time, specimens for histomorphometry were sampled from the distal stump. After sampling, wounds were closed. Four days later, the animals were sacrificed by perfusion with formalin, and the spinal cord and dorsal root ganglia were harvested.

\section{Surgical Procedure}

Surgical procedures were performed by a single operator (M.H.) using standard microsurgical techniques under an operating microscope (Varioskop, Zeiss). The sciatic trunk was exposed on the right side via a transgluteal approach at the posterolateral aspect of the thigh and through the interval between the superficial gluteal muscle and the biceps femoris muscle. In all rat groups, a segment of the sciatic nerve was excised just distal to the sciatic notch and removed to create a $15-\mathrm{mm}$ defect. In the negative control Group A, there was no repair and the nerve stumps were left in situ. In Group B, the defect was reconstructed using an isogenic mixed fascicular group harvested from a donor animal. This fascicular group was obtained by interfascicular neurolysis from the sciatic nerve. In Group C, a segment of the saphenous nerve was used for reconstruction. The animals of Group D received the motor branch of the femoral nerve as graft. To obtain the grafts for Groups C and D, a groin incision was made to expose the femoral neurovascular bundle. In the experimental Group E, a fascicular group was dissected from the sciatic nerve, excised over a length of $16 \mathrm{~mm}$ directly distal to the gap, and inserted into the gap (Fig. 3). Nerve coaptation was performed with two 10-0 epineural sutures for each suture site (Fig. 4).

\section{Functional Muscle Testing}

For the analysis of muscle force, the sciatic nerve was exposed. Proximal to the point of reconstruction, a cuff electrode was placed around the sciatic nerve. To obtain the force of muscles innervated by the TIB as well as the 


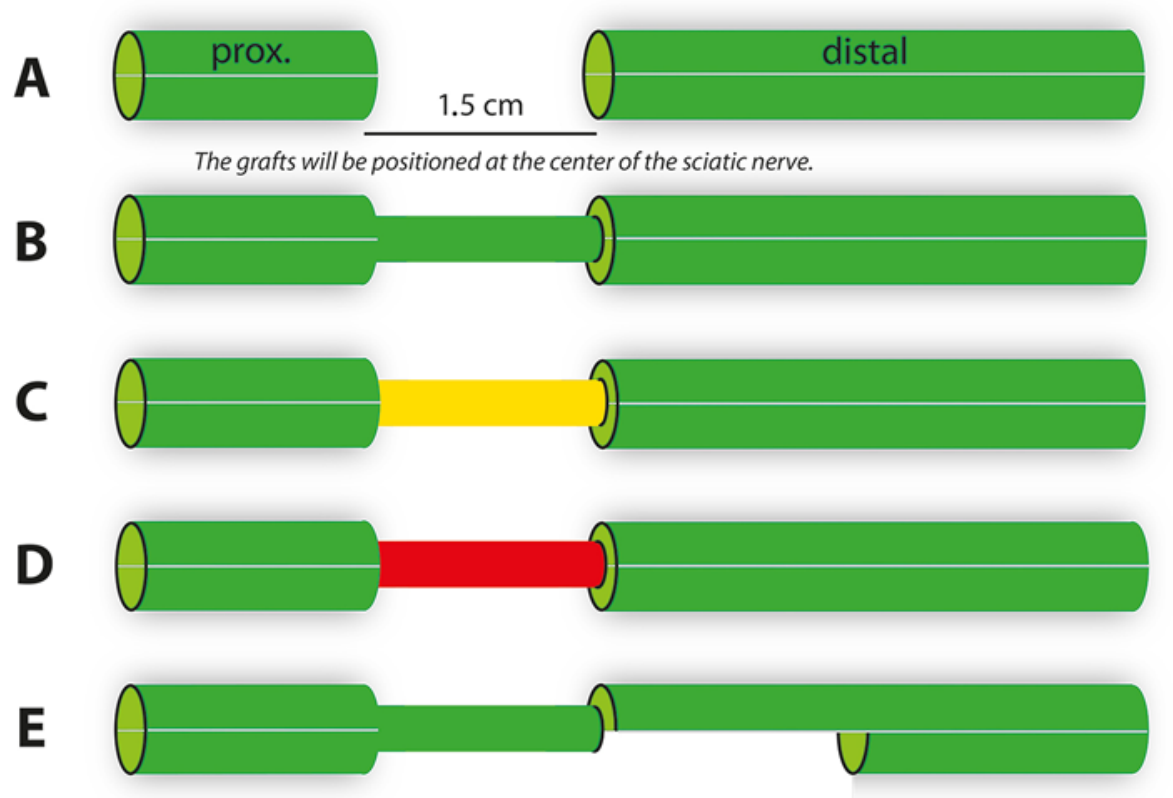

Group A: $n=5$

gap without reconstruction

Group B: $n=10$

reconstruction using a mixed fascicle

Group C: $n=10$ reconstruction using a sensory nerve

Group D: $n=10$ reconstruction using a motor nerve

Group E: $n=10$ reconstruction using a fascicular shift

FIG. 1. Study design as regards nerve grafting technique. Drawing of 5 segments of the sciatic nerve with a 15 -mm nerve defect and the group-specific nerve reconstruction plan. The thin gray line indicates the 2 fascicles of the sciatic nerve (tibial and common peroneal fascicle). The sural nerve is not shown. Forty-five Lewis rats were randomized into 5 groups. Ten additional rats were used to harvest the required donor nerves. $n=$ number of rats; prox. = proximal. Figure is available in color online only.

$\mathrm{CP}$, the distal tendon of the tibialis anterior muscle and the gastrocnemius muscle, respectively, were exposed and connected to the force transducer consecutively. The muscles were assessed by supramaximal nerve stimulation. The pulse duration was fixed at $0.1 \mathrm{msec}$, with constant voltage. To determine the maximal force, the individual stimulation parameters were identified iteratively $(0.1-10$ $\mathrm{mA}, 10-100 \mathrm{~Hz}$ ). The signals generated by the force transducer were passed through an amplifier and displayed on an oscilloscope.

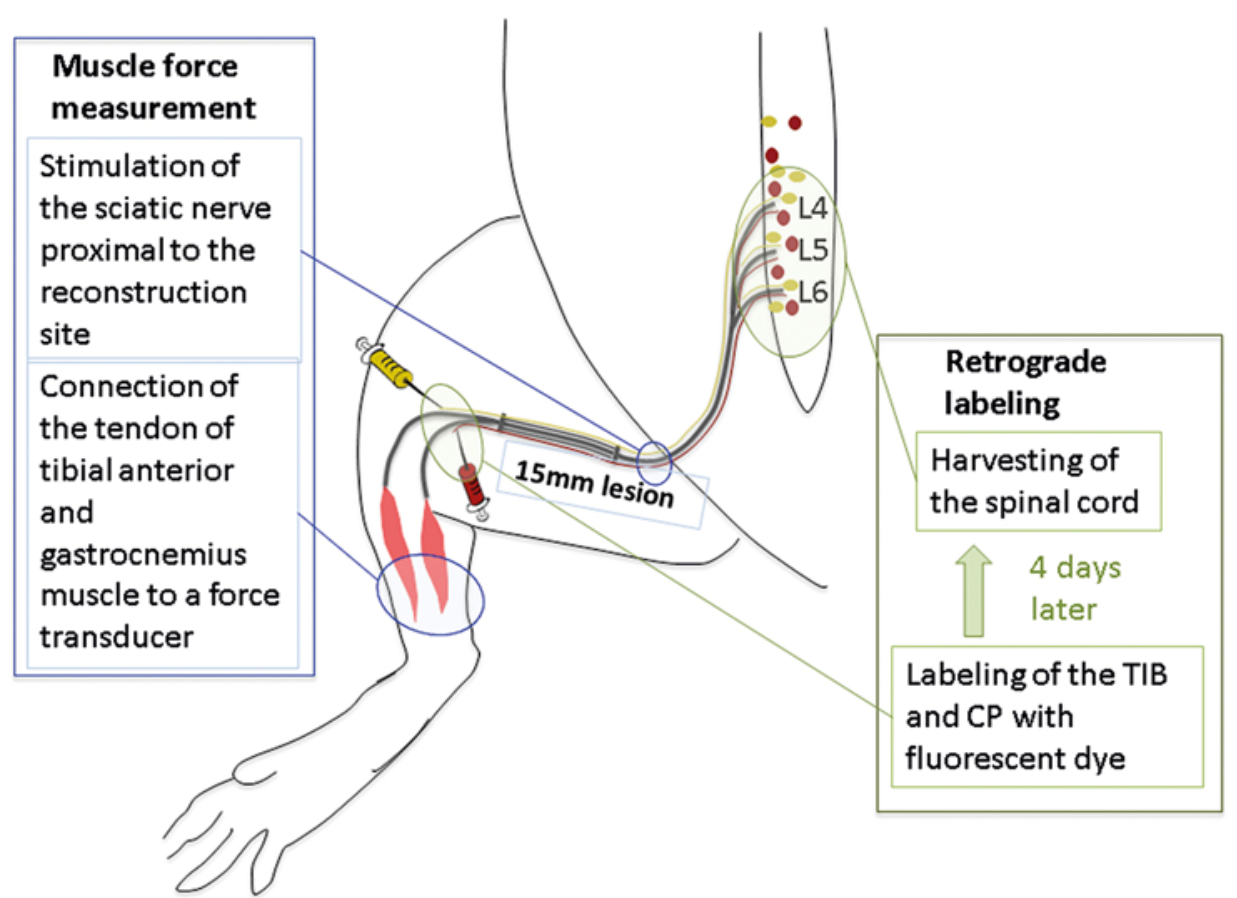

FIG. 2. Experimental setup for muscle force measurement and retrograde labeling. Twelve weeks after surgery, nerve regeneration was assessed with muscle force measurement and subsequent retrograde labeling. $\mathrm{CP}=$ common peroneal nerve; $\mathrm{TIB}=$ tibial nerve. Figure is available in color online only. 


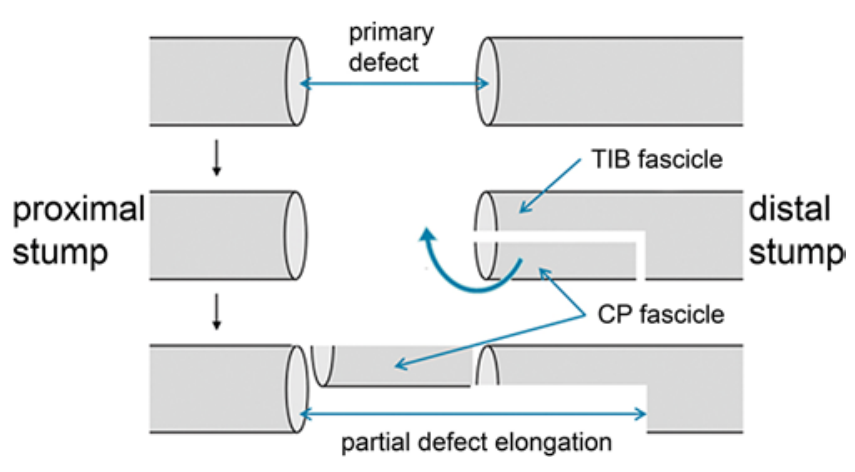

FIG. 3. Drawing of the FSP. A fascicular group of the nerve distal to the injury is harvested at an appropriate length to bridge the nerve defect. The effect of the partial defect elongation on nerve regeneration was also investigated. Figure is available in color online only.

\section{Histomorphometry}

After the muscle force analysis, the sciatic nerve was separated from surrounding tissue to inspect the whole reconstruction site. Distal to the injury site, the TIB and $\mathrm{CP}$ were transected. Nerve biopsy specimens of the TIB and $\mathrm{CP}$ were harvested, immediately immersed in a $2.5 \%$ solution of glutaraldehyde buffered with cacodylate, stained with $4 \%$ osmium tetroxide, and embedded in epoxy resin. Semi-thin sections were obtained using an ultramicrotome (Leica). The sections were stained with $1 \%$ paraphenylenediamine in absolute alcohol and examined under light microscopy (Nikon). The morphometric measurements of all sections were performed using a semiautomatic image analyzing system (NIS Elements). Gross morphometric measurements were obtained at a magnification of $\times 20$; detailed measurements, at a magnification of $\times 60$. To minimize operator-dependent errors, only one person analyzed the cross sections.

\section{Retrograde Labeling}

For the simultaneous double labeling, the transected proximal nerve stumps were put into a Vaseline well filled with Fluoro-Gold or Fluoro-Ruby. To avoid bias due to the different penetration of the stains, the Fluoro-Gold and Fluoro-Ruby were alternated from one animal to the next, meaning the TIB was gold for every second animal and red for every other. After 1 hour of absorbing the stains, the Vaseline wells were removed and all wounds were closed. After 4 days to allow retrograde transport, the animals were anesthetized and perfused with 150 $\mathrm{ml}$ sodium chloride and $150 \mathrm{ml} 4 \%$ paraformaldehyde (PFA) solution. The spinal cord was harvested and fixed in 4\% PFA for 24 hours. The specimens were postfixed with $30 \%$ sucrose buffered in 4\% PFA overnight. Spinal cord segments were embedded in tissue freezing medium (Tissue-Tek). Using a freezing microtome (Leica), we cut the spinal cords into $20-\mu \mathrm{m}$ sections. Motor neurons were quantified under an epifluorescence microscope (Nikon) at a magnification of $\times 40$ (Fig. 5). To minimize operatordependent errors, only one person analyzed the cross sections.

\section{Statistical Analysis}

Descriptive statistics were obtained for motor neuron pool numbers, histomorphometric data, and functional muscle evaluation. With a nonparametric test, the samples were tested for normality of distribution (maximum likelihood method for estimating the mean $[\mu]$ and standard deviation [б], Kolmogorov-Smirnov test for assessing goodness of fit). The samples showed homogeneity of variance.
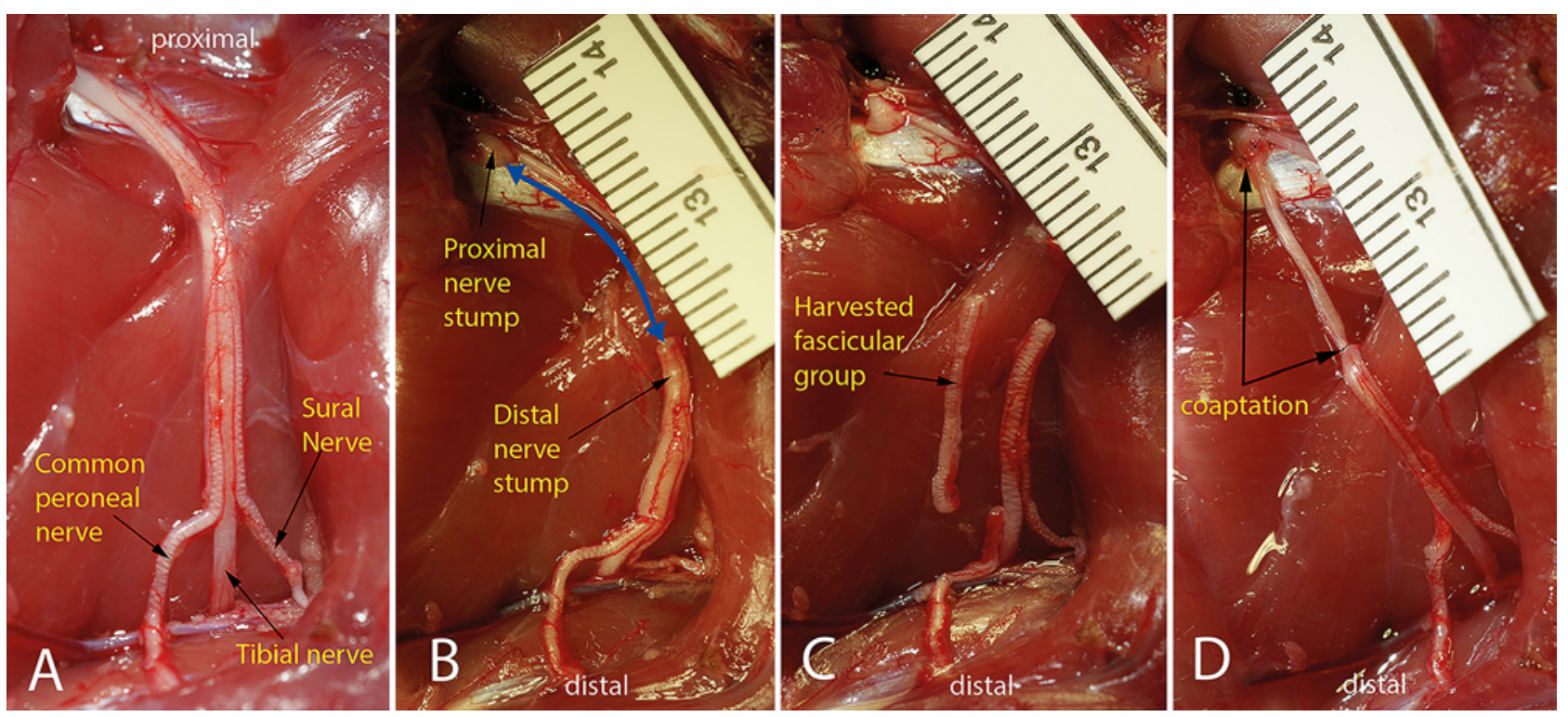

FIG. 4. Intraoperative documentation of the FSP. A: Operative Step 1. The sciatic nerve and its 3 fascicles (TIB, CP, and sural nerve) are dissected. B: Operative Step 2. A 15-mm defect is created. C: Operative Step 3. Distal to the nerve defect, a fascicular group is harvested at the required length. D: Operative Step 4. The common peroneal fascicle is harvested, shifted, and used to bridge the nerve defect. Figure is available in color online only. 

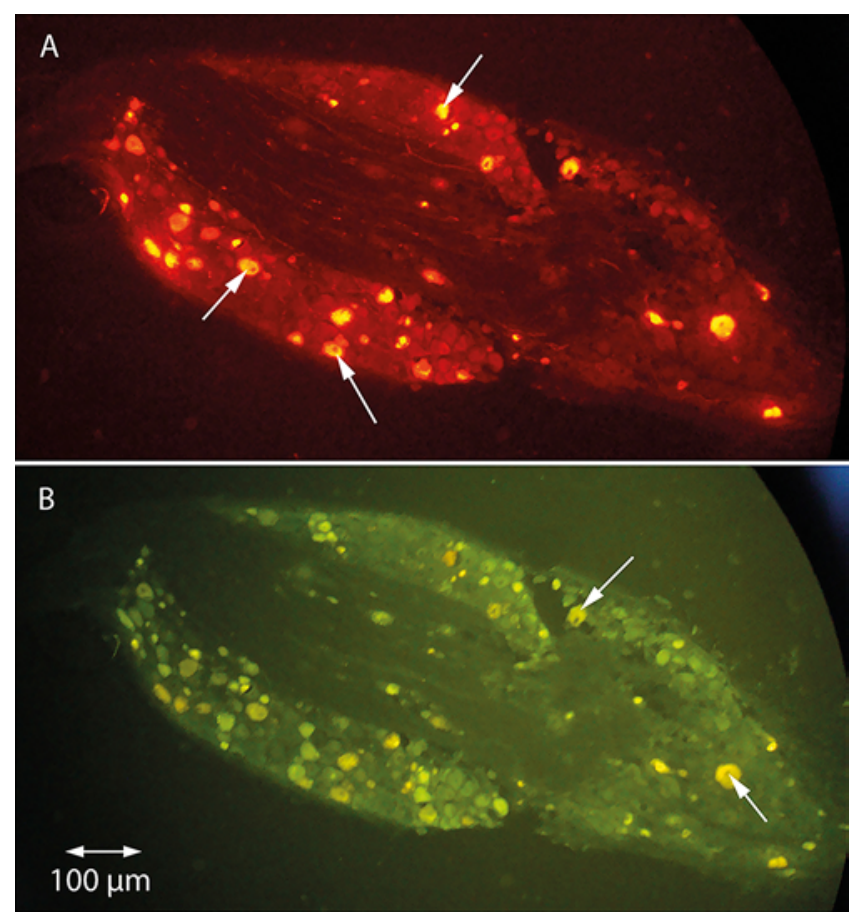

FIG. 5. Retrograde labeling of the dorsal root ganglia under epifluorescence microscopy. A: Dorsal root ganglia labeled with Fluoro-Ruby. B: Dorsal root ganglia labeled with Fluoro-Gold. Regeneration in both the TIB and the CP is identified individually by simultaneous double labeling. Arrows indicate the labeled sensory neurons. Figure is available in color online only.

A 1-way ANOVA and post hoc test were performed. Correlations (r) among motor neuron number, histomorphometric data, and functional muscle evaluation were evaluated using Pearson's correlation. For statistical analysis, the SigmaStat software package (SPSS Inc.) was used. A $\mathrm{p}$ value of 0.05 was significant.

\section{Results}

\section{Retrograde Labeling}

In retrograde labeling, the total amount of regenerated, stain-labeled neurons of the spinal cord was taken into account (Fig. 6). The counts of Groups B (1490.43 \pm 794.80$)$, D $(1720.00 \pm 866.421)$, and E $(1958.75 \pm 657.21)$ were statistically equivalent $(p<0.95)$. In comparison with the level in experimental Group E, neuronal regeneration in Group C (1263.50 \pm 538.90) was reduced by one-third. Given the small sample sizes of the groups, this difference was found to represent a statistically verified tendency at a level of $p<0.1$. In the negative control Group A, spontaneous nerve regeneration was very diffuse without the development of bundles, so that precise staining was not feasible.

\section{Functional Muscle Testing}

Muscle force measurement (Fig. 7) showed an impressive functional advantage after reconstruction using a mixed graft compared with a sensory graft. Regarding the tibialis anterior muscle (and with it the regeneration of the CP), Group B $(22.68 \pm 12.67 \mathrm{mN})$ achieved a 3 -fold increased maximal force compared with Group C (10.22 \pm $8.80 \mathrm{mN}$ ). This was a significant difference at a level of $\mathrm{p}$ $<0.05$. Groups D $(17.80 \pm 14.89 \mathrm{mN})$ and $\mathrm{E}(19.97 \pm 13.01$ $\mathrm{mN}$ ) were in the upper midfield and at a significance level of $p<0.01$ were superior to Group A. The same trend as described in retrograde labeling was found in functional muscle testing. The muscle force of Group $\mathrm{E}$ was superior to that in the sensory Group $\mathrm{C}$ at a level of $\mathrm{p}<0.1$. The negative control Group A showed minimal force measure-

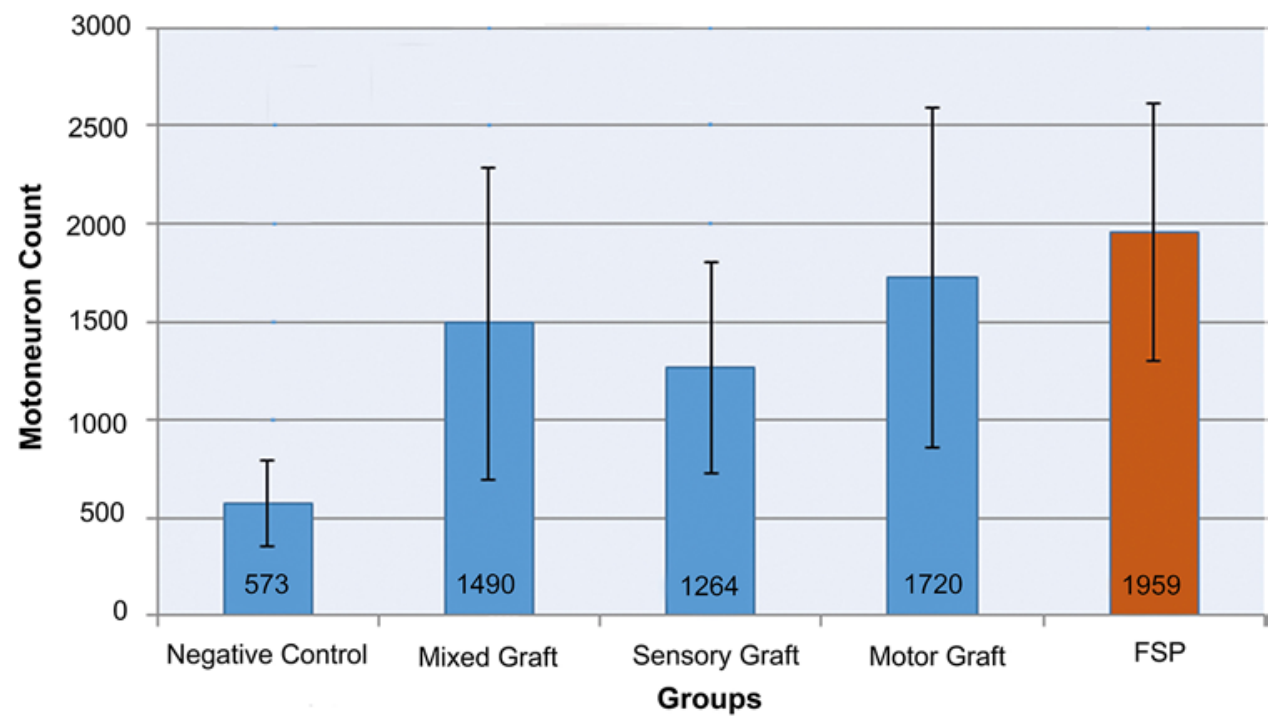

FIG. 6. Results of retrograde labeling showing the mean motor neuron counts (total amount of stain-labeled neurons in the spinal cord). The experimental Group E (FSP) performed significantly better $(p<0.05)$ than the negative control Group A and was statistically equivalent to Group B (mixed graft). Motor neurons in Group C (sensory graft) were reduced by one-third compared with levels in Group $E$, which represents a statistically verified tendency at a level of $p<0.1$. Error bars indicate the standard deviation. Figure is available in color online only. 


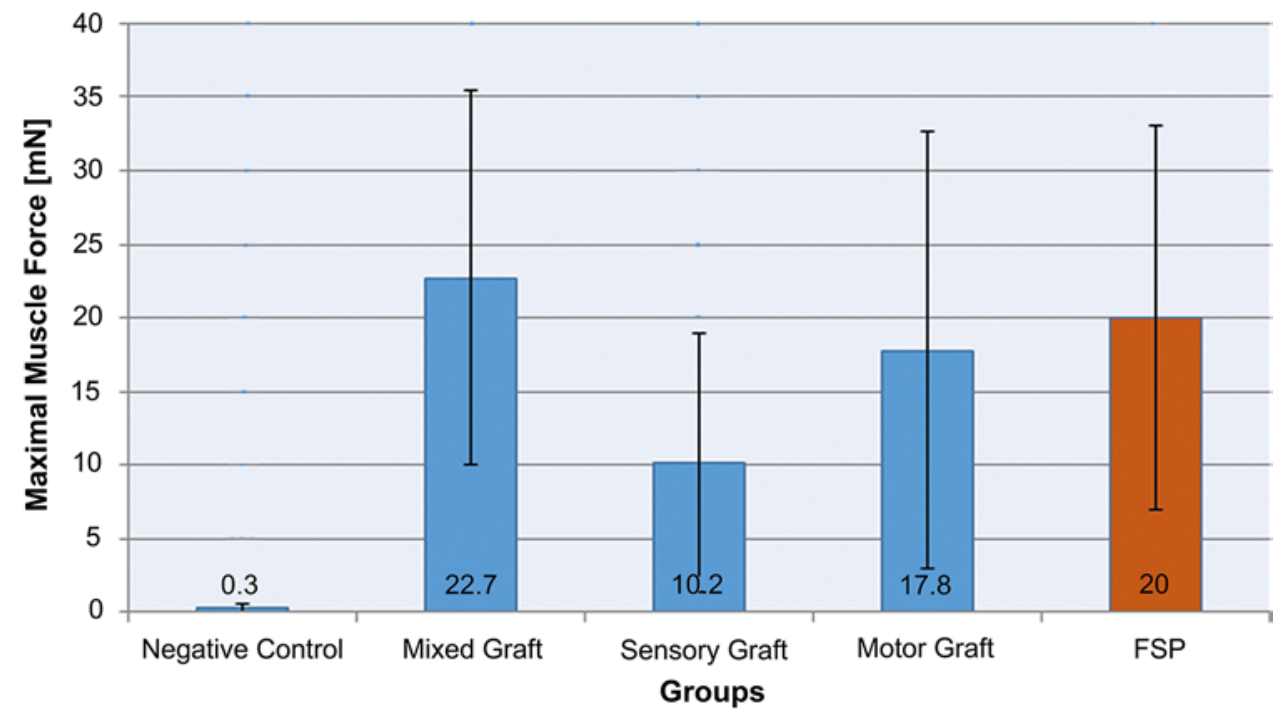

FIG. 7. Results of mean maximal muscle force of the tibialis anterior muscle. The experimental Group E (FSP) performed significantly better $(p<0.01)$ than the negative control Group A and was statistically equivalent to Group B (mixed graft). Maximal muscle force in Group C (sensory graft) was reduced compared with that in Group E, which represents a statistically verified tendency at a level of $p<0.1$. Error bars indicate the standard deviation. Figure is available in color online only.

ments that were of no functional value $(0.34 \pm 0.56 \mathrm{mN})$. Two animals in Group A and 1 animal in Group C showed complete denervation of the tibialis anterior muscle.

\section{Histomorphometry}

Tables 1 and 2 show the quantitative results of the histomorphometric analysis. In summary, all reconstructed groups revealed successful regeneration for both the TIB and CP with no statistically significant differences.

\section{Detailed Comparison of the Mixed Graft and the Fascicular Shift}

Group B (mixed graft) was compared with Group E (FSP) to evaluate the effect of the partial defect elongation induced by harvesting a fascicular group distal to the repair site (Fig. 8). Although the regenerating CP in Group E had one more barrier to overcome than in Group B (namely the site of fascicle harvesting distal to the primary nerve defect), the outcome for the CP was equivalent in the 2 groups. None of the analyses revealed impaired nerve re-

TABLE 1. Histomorphometric analyses of the $C P$ in the different experimental groups

\begin{tabular}{ccccc}
\hline & \multicolumn{4}{c}{ Group } \\
\cline { 2 - 5 } CP & $\begin{array}{c}\text { B, Mixed } \\
\text { Graft }\end{array}$ & $\begin{array}{c}\text { C, Sensory } \\
\text { Graft }\end{array}$ & $\begin{array}{c}\text { D, Motor } \\
\text { Graft }\end{array}$ & $\begin{array}{c}\text { E, } \\
\text { FSP }\end{array}$ \\
\hline $\begin{array}{c}\text { No. of axons } \\
\left(\times 10^{3}\right)\end{array}$ & $2.45 \pm 0.56$ & $1.49 \pm 1.09$ & $1.60 \pm 0.85$ & $3.24 \pm 1.98$ \\
\hline $\begin{array}{c}\text { Fiber density } \\
\left(\times 10^{3}\right)\end{array}$ & $21.7 \pm 8.40$ & $16.3 \pm 4.20$ & $26.7 \pm 14.8$ & $22.8 \pm 8.53$ \\
\hline G-ratio & $0.37 \pm 0.19$ & $0.12 \pm 0.03$ & $0.41 \pm 0.15$ & $0.56 \pm 0.20$ \\
\hline
\end{tabular}

There is no statistically significant difference between the reconstruction groups $(B-E)$ in this analysis. generation. Both branches of the sciatic nerve were regenerating, although the common peroneal fascicle was used for reconstruction in the FSP.

\section{Discussion}

Peripheral nerves are the gateway between the brain and the end organs in the body; they provide routes for both sensory and motor information to be relayed. When these pathways are damaged, severe loss of function occurs. Nerve reconstruction has been attempted using nerve grafts since the late 19th century ${ }^{9}$ but with limits in both quantity and quality. We were able to show in this study that the FSP is a successful alternative to nerve reconstruction, providing both a sufficient amount and quality of nerve tissue. This is demonstrated through our investigation of the different aspects of nerve regeneration and our comparison of the FSP method with the other established methods of nerve repair including sensory, motor, or mixed grafting.

Early 20th century attempts at nerve grafting focused

TABLE 2. Histomorphometric analyses of the TIB in the different experimental groups

\begin{tabular}{ccccc}
\hline & \multicolumn{4}{c}{ Group } \\
\cline { 2 - 5 } TIB & $\begin{array}{c}\text { B, Mixed } \\
\text { Graft }\end{array}$ & $\begin{array}{c}\text { C, Sensory } \\
\text { Graft }\end{array}$ & $\begin{array}{c}\text { D, Motor } \\
\text { Graft }\end{array}$ & $\begin{array}{c}\text { E, } \\
\text { FSP }\end{array}$ \\
\hline $\begin{array}{c}\text { No. of axons } \\
\left(\times 10^{3}\right)\end{array}$ & $3.85 \pm 2.17$ & $3.80 \pm 4.07$ & $1.91 \pm 1.36$ & $3.27 \pm 2.00$ \\
\hline $\begin{array}{c}\text { Fiber density } \\
\left(\times 10^{3}\right)\end{array}$ & $13.0 \pm 8.14$ & $25.4 \pm 2.41$ & $11.1 \pm 5.66$ & $20.6 \pm 7.57$ \\
\hline G-ratio & $0.29 \pm 0.15$ & $0.48 \pm 0.23$ & $0.52 \pm 0.09$ & $0.45 \pm 0.18$ \\
\hline
\end{tabular}

There is no statistically significant difference between the reconstruction groups $(B-E)$ in this analysis. 


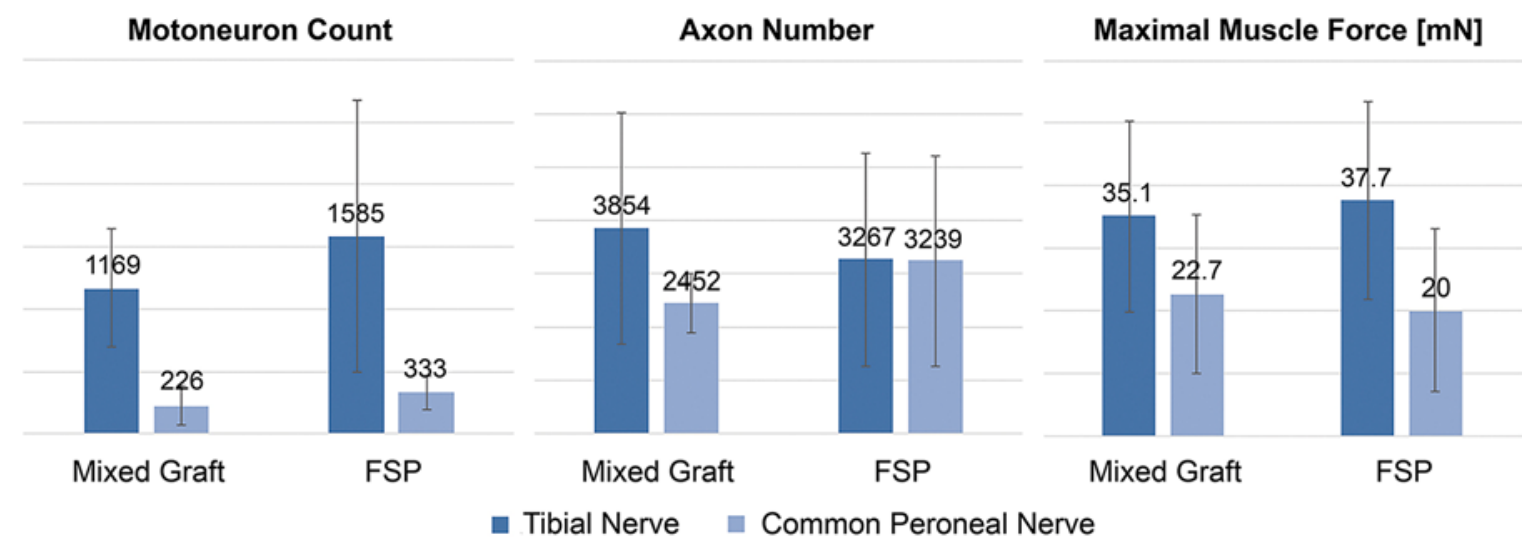

FIG. 8. Detailed comparison of Group B (mixed graft) and Group E (FSP). Equal results between the mixed graft and the fascicular shift indicate that harvesting a nerve graft distal to the nerve injury does not compromise nerve regeneration. Figure is available in color online only.

on saving viable nerve tissue while transplanting single fascicles, with limited functional outcomes.,17,20,33,36 While these early studies were not successful, investigators recognized that conserving healthy nerve tissue was critical to the final nerve repair. After both world wars, which required increased creativity in peripheral nerve surgery, new ways of nerve transplantation were explored, leading to more effective and sophisticated approaches of nerve grafting. ${ }^{10,26}$ Unfortunately, at the time there were no structured studies, and most reports consisted of single-case studies that provided no basis for further investigations. MacCarty, for instance, bridged a lesion of the sciatic nerve by shifting a fascicular group harvested proximally from the primary defect. ${ }^{19}$ This 2 -staged procedure resulted in successful regeneration, but with a prolonged healing process.

During the second half of the 20th century, the research focus shifted toward artificial grafts. ${ }^{8,21,31,34,35}$ However, these methods have not been clinically successful for long defects either, ${ }^{8,31}$ and the problem of nerve reconstruction remained unchanged, namely limited available transplant material. This is a particular problem in large proximal nerve defects, which are difficult to reconstruct due to the major cross-section dimension and the length of the defect. However, modern microsurgical techniques and increased understanding of the biology of nerve regeneration have improved the manner in which nerve grafting is performed. ${ }^{6,26,39}$

Maria Siemionow was the first to restart research into single-fascicle grafting and applied this paradigm in the first structured rat models. ${ }^{30,32}$ This research demonstrated that single-fascicle grafting was a feasible method of nerve reconstruction that allowed reduced donor-site morbidity and increased material availability. ${ }^{27}$ Subsequently, Koshima et al. successfully showed that single fascicles from the proximal and distal stump of the injured nerve could be used to reconstruct a nerve gap. ${ }^{16}$

The study presented here is a continuation of our previous work demonstrating that a single fascicle provides enough guidance for successful regeneration within the sensory system. ${ }^{37}$ This finding is also reflected in the histomorphometric data of the present study, revealing no statistical difference in fiber counts among all reconstructed groups. Even though the concept of specific motor and sensory Schwann cells is disputed, data from both our previous study and the present study clearly demonstrate that motor axons are more likely to regenerate in the same physical and trophic environment as that provided by a modality-matched graft. Furthermore, there are ample data indicating that these entities do indeed exist and that modality-matched grafts do make a difference in nerve regeneration. ${ }^{1-4,21,22}$ The FSP provides such grafts harvested directly from the distal segment of the injured nerve, thus bypassing 2 difficulties at once: the sacrifice of a second nerve and mismatched-modality grafting.

The main finding of our study is that the FSP is superior to the gold standard of sensory grafting in terms of nerve regeneration. This finding has been confirmed in two ways. First, the motor neuron counts prove a numerically superior regeneration with the FSP as compared with sensory nerve grafting. Second, the muscle force measurements indicate impairment of the neuromuscular unit following sensory grafting, with deferred excitability and reduced maximal force. Furthermore, the FSP not only provided qualitatively adequate donor grafts, but also a high number of axons, which likely contributes to the good regeneration properties. This is reflected by the histomorphometric data (Tables 1 and 2.).

A critical issue was the assessment of potential donorsite morbidity induced by the partial defect elongation. From a surgeon's perspective, the most important objective is to preserve healthy tissue to achieve optimal recovery of motor function. Therefore, taking healthy tissue from the distal nerve to repair the original deficit may be viewed as simply creating another deficit in a different location. Our results show this to be false. Firstly, the muscle force and the motor neuron counts after the FSP were equivalent to those of the mixed nerve graft. This is direct evidence that the manipulation of the distal nerve stump did not hinder regeneration. Secondly, as was demonstrated by all investigations, continuity of nerve repair was restored in both the TIB and the CP after the FSP. This phenomenon can be explained by the concept of side-to-side repair, showing that nerve regenerates alongside its healthy nerve graft 
and is finally capable of deviating into the distal portion of a loose nerve end. ${ }^{23}$ This suggests that inherent physiological processes use the transplanted graft as a guide for nerve regeneration.

Current understanding of nerve regeneration suggests that normal nerve architecture distal to the defect is dissolved so that the absence of one fascicle or even an entire fascicle group does not further impair regeneration. The finding that continuous nerve regeneration occurred even after a portion of the distal stump was translocated highlights the need to review the role of the distal nerve stump as a signal source and a structural matrix in the process of nerve regeneration.

As the distal stump of an injured nerve undergoes Wallerian degeneration, nerve regeneration does not only mean rewiring nerve fibers, but also renewing the entire distal axonal pathway. This is a highly complex process, as reviewed elsewhere. ${ }^{11}$ Even after a nerve injury of the axonotmesis type (Sunderland II), where the perineurium and/or endoneurium are preserved and only the continuity of the axons is lost, nerve regeneration still results in a defect that heals with reduced nerve conduction velocity. ${ }^{25}$ Responsible for this impairment is a reorganization process that leads to shortened internodal segments, the formation of mini-fascicles, the development of scar tissue, and a decreased diameter of the axons. Regardless of the severity of the injury, the endoneurium together with its surrounding Schwann cells acts as a consistent structural matrix and signal source, shepherding axonal regeneration around the formation of scar tissue. After the ingrowth of axons, reactive Schwann cells migrate and form new myelin sheaths surrounding the axons, completing the regeneration process. The FSP takes advantage of this inherent physiological process by maintaining the presence of Schwann cells and a portion of the endoneurium to successfully guide the regeneration of nerve fibers. Finally, it has been suggested that axons need a type-specific (sensory/motor) physical and humoral environment that is provided by specific motor/sensory Schwann cells, which would also be provided in our reconstructive paradigm. In standard brachial plexus repair, "predegenerated nerves or nerve segments" distal to the lesion are often used. This may be the medial brachial and/or antebrachial cutaneous nerves and, in cases of lower root avulsions, the entire ulnar nerve. In fact, the concept of predegenerated nerve grafts with activated Schwann cells and empty tubes has been suggested to improve rather than hinder regeneration. ${ }^{6,15,29}$ Finally, there is ample evidence that reactive Schwann cells and macrophage activity play an essential role in nerve regeneration..$^{12,14,28}$

In summary, this experimental study shows that a nerve segment distal to the injury can provide graft material to reconstruct a defect. A limitation of this study was the standard deviations of the muscle force measurements, which would have probably provided significant results with a larger sample size. In this study we investigated acute peripheral nerve injuries in rats, not delayed injuries that lead to muscular atrophy and not human nerve injuries. Hence, further clinical studies may be required to verify our promising results. We believe that the approach of using a fascicular group as a graft has the potential to reduce donor-site morbidity as well as the problem of limited availability of grafting material and provide modalitymatched grafting.

\section{Conclusions}

Overall, the idea of the FSP is to "shift" one fascicular group from a distal segment of the injured nerve to the defect site. This procedure has 2 main benefits: First, local harvesting of the transplant avoids affecting another nerve. Second, the composition of motor and sensory fibers is maintained. Thus, any quantitative and qualitative mismatch between the recipient nerve and the transplant is avoided, and the supreme principle of reconstructive surgery is achieved, that is, to replace like with like. The main finding of this experimental study is that the FSP is statistically superior to a standard sensory graft in terms of motor recovery. Furthermore, manipulation of the distal nerve stump does not interfere with nerve regeneration. Therefore, the FSP can be an attractive alternative in the reconstruction of large proximal nerve defects, as is often required in brachial plexus surgery, or in the reconstruction of nerve trunk defects.

\section{Acknowledgments}

This study was exclusively funded by the Christian Doppler Research Foundation of the Austrian Council for Research and Technology Development.

\section{References}

1. Brenner MJ, Hess JR, Myckatyn TM, Hayashi A, Hunter DA, Mackinnon SE: Repair of motor nerve gaps with sensory nerve inhibits regeneration in rats. Laryngoscope 116:16851692,2006

2. Brushart TM: Motor axons preferentially reinnervate motor pathways. J Neurosci 13:2730-2738, 1993

3. Brushart TM: Preferential motor reinnervation: a sequential double-labeling study. Restor Neurol Neurosci 1:281-287, 1990

4. Brushart TM: Preferential reinnervation of motor nerves by regenerating motor axons. J Neurosci 8:1026-1031, 1988

5. Chentanez V, Cha-oumphol P, Kaewsema A, Agthong S, Huanmanop T: Accuracy of the three-window sampling method in morphometric analysis of human sural nerve. $\mathbf{J}$ Neurosci Methods 157:154-157, 2006

6. Dahlin L, Johansson F, Lindwall C, Kanje M: Chapter 28: Future perspective in peripheral nerve reconstruction. Int Rev Neurobiol 87:507-530, 2009

7. Davis L, Cleveland DA: Experimental studies in nerve transplants. Ann Surg 99:271-283, 1934

8. de Ruiter GC, Malessy MJ, Yaszemski MJ, Windebank AJ, Spinner RJ: Designing ideal conduits for peripheral nerve repair. Neurosurg Focus 26(2):E5, 2009

9. Dellon ES, Dellon AL: The first nerve graft, Vulpian, and the nineteenth century neural regeneration controversy. J Hand Surg Am 18:369-372, 1993

10. Eberhard D, Millesi H: Split nerve grafting. J Reconstr Microsurg 12:71-76, 1996

11. Fox IK, Brenner MJ, Johnson PJ, Hunter DA, Mackinnon SE: Axonal regeneration and motor neuron survival after microsurgical nerve reconstruction. Microsurgery 32:552-562, 2012

12. George R, Griffin JW: Delayed macrophage responses and myelin clearance during Wallerian degeneration in the central nervous system: the dorsal radiculotomy model. Exp Neurol 129:225-236, 1994 
13. Guillen J: FELASA guidelines and recommendations. J Am Assoc Lab Anim Sci 51:311-321, 2012

14. Höke A, Redett R, Hameed H, Jari R, Zhou C, Li ZB, et al: Schwann cells express motor and sensory phenotypes that regulate axon regeneration. J Neurosci 26:9646-9655, 2006

15. Kerns JM, Danielsen N, Holmquist B, Kanje M, Lundborg G: The influence of predegeneration on regeneration through peripheral nerve grafts in the rat. Exp Neurol 122:28-36, 1993

16. Koshima I, Narushima M, Mihara M, Uchida G, Nakagawa M: Fascicular turnover flap for nerve gaps. J Plast Reconstr Aesthet Surg 63:1008-1014, 2010

17. Létiévant JJE: Traité des sections nerveuses; physiologie pathologique, indications, procédés opératoires. Paris: J.B. Baillière, 1873

18. Luís AL, Amado S, Geuna S, Rodrigues JM, Simões MJ, Santos JD, et al: Long-term functional and morphological assessment of a standardized rat sciatic nerve crush injury with a non-serrated clamp. J Neurosci Methods 163:92-104, 2007

19. MacCarty CS: Two-stage autograft for repair of extensive damage to sciatic nerve. Report of case. J Neurosurg 8:319_ 322,1951

20. Mackenzie KA: Resection of the sciatic nerve. Neuroplasty: end results. Ann Surg 50:295-312, 1909

21. Mackinnon SE: Surgical management of the peripheral nerve gap. Clin Plast Surg 16:587-603, 1989

22. Madison RD, Robinson GA, Chadaram SR: The specificity of motor neurone regeneration (preferential reinnervation). Acta Physiol (Oxf) 189:201-206, 2007

23. McCallister WV, Cober SR, Norman A, Trumble TE: Using intact nerve to bridge peripheral nerve defects: an alternative to the use of nerve grafts. J Hand Surg Am 26:315-325, 2001

24. Meek MF, Coert JH: Clinical use of nerve conduits in peripheral-nerve repair: review of the literature. J Reconstr Microsurg 18:97-109, 2002

25. Muratori L, Ronchi G, Raimondo S, Giacobini-Robecchi MG, Fornaro M, Geuna S: Can regenerated nerve fibers return to normal size? A long-term post-traumatic study of the rat median nerve crush injury model. Microsurgery 32:383387, 2012

26. Narakas A, Verdan C: [Nerve grafts.] Z Unfallmed Berufskr 62:137-152, 1969 (Fr)

27. Okuyama N, Nakao Y, Takayama S, Toyama Y: Effect of number of fascicle on axonal regeneration in cable grafts. Microsurgery 24:400-407, 2004

28. Rodríguez FJ, Gómez N, Labrador RO, Butí M, Ceballos D, Cuadras J, et al: Improvement of regeneration with predegenerated nerve transplants in silicone chambers. Restor Neurol Neurosci 14:65-79, 1999

29. Rodríguez FJ, Verdú E, Ceballos D, Navarro X: Nerve guides seeded with autologous Schwann cells improve nerve regeneration. Exp Neurol 161:571-584, 2000

30. Scharpf J, Meirer R, Zielinski M, Unsal M, Ramineni P, Nair $\mathrm{D}$, et al: A novel technique for peripheral nerve repair. Laryngoscope 113:95-101, 2003

31. Siemionow M, Brzezicki G: Current techniques and concepts in peripheral nerve repair. Int Rev Neurobiol 87:141-172, 2009
32. Siemionow M, Zielinski M, Meirer R: The single-fascicle method of nerve grafting. Ann Plast Surg 52:72-79, 2004

33. Stookey B: The futility of bridging nerve defects by means of nerve flaps. Surg Gynecol Obstet 29:287-311, 1919

34. Strauch B: Use of nerve conduits in peripheral nerve repair. Hand Clin 16:123-130, 2000

35. Strauch B, Rodriguez DM, Diaz J, Yu HL, Kaplan G, Weinstein DE: Autologous Schwann cells drive regeneration through a 6-cm autogenous venous nerve conduit. J Reconstr Microsurg 17:589-597, 2001

36. Tillmanns H: Ueber die operative Behandlung von Substanzverlusten an peripheren Nerven. Berlin: Arch klin Chir 32:923-946, 1885

37. Tzou $\mathrm{CH}$, Aszmann $\mathrm{OC}$, Frey M: Bridging peripheral nerve defects using a single-fascicle nerve graft. Plast Reconstr Surg 128:861-869, 2011

38. Varejão AS, Cabrita AM, Meek MF, Bulas-Cruz J, MeloPinto P, Raimondo S, et al: Functional and morphological assessment of a standardized rat sciatic nerve crush injury with a non-serrated clamp. J Neurotrauma 21:1652-1670, 2004

39. Zochodne DW: The challenges and beauty of peripheral nerve regrowth. J Peripher Nerv Syst 17:1-18, 2012

\section{Disclosures}

The authors report no conflict of interest concerning the materials or methods used in this study or the findings specified in this paper.

\section{Author Contributions}

Conception and design: Aszmann, Hader. Acquisition of data: Hader, Sporer, Unger, Wakolbinger. Analysis and interpretation of data: Hader, Sporer, Unger, Wakolbinger. Drafting the article: Hader, Sporer, Roche. Critically revising the article: Aszmann, Roche, Bergmeister, Wakolbinger. Reviewed submitted version of manuscript: Aszmann, Sporer, Bergmeister. Approved the final version of the manuscript on behalf of all authors: Aszmann. Statistical analysis: Hader. Administrative/technical/material support: Sporer, Unger. Study supervision: Aszmann.

\section{Supplemental Information}

\section{Previous Presentations}

Portions of this work were presented as oral presentations at the 56th Austrian Surgery Convention held in Linz, Austria, on June 5, 2015; the 53rd Annual Convention of the ÖGPÄRC held in Salzburg, Austria, on September 18, 2015; and the 37th Annual Conference of the German-Speaking Association of Microsurgery held in Bochum, Germany, on December 5, 2015.

\section{Correspondence}

Oskar C. Aszmann, Division of Plastic and Reconstructive Surgery, Department of Surgery, Medical University of Vienna, Währinger Gürtel 18-20, Vienna 1090, Austria. email: oskar. aszmann@meduniwien.ac.at. 